\title{
Fascículo Distal del Ligamento Tibiofibular Anterior: Presencia, Biometría y Relación con la Tróclea Talar
}

\author{
Distal Fascicle of The Anterior Tibiofibular Ligament: \\ Presence, Biometric Characteristics and Relation with the Talar Trochlea
}

Cabezas, J. J. ${ }^{1,2} \&$ Olave, E. ${ }^{3}$

CABEZAS, J. J. \& OLAVE, E. Fascículo distal del ligamento tibiofibular anterior: Presencia, biometría y relación con la tróclea talar. Int. J. Morphol., 38(2):472-476, 2020.

RESUMEN: El ligamento tibiofibular anterior suele presentar un fascículo distal independiente de la banda principal, denominado fascículo distal del ligamento tibiofibular anterior (FD-LTFA). Este discurre oblicuamente, cubriendo sus fibras más inferiores la zona anterolateral de la articulación talocrural. Su presencia se asocia a pellizcamiento del tobillo en esta zona, el cual puede producir un desgaste cartilaginoso de la cara anterolateral de la tróclea talar. El propósito de este estudio fue determinar la presencia y biometría del FD-LTFA, y su relación con la troclea talar en tobillos de un grupo de individuos Chilenos. En este estudio se utilizaron 30 miembros inferiores de cadáveres de individuos adultos. Se evaluó en el fascículo distal: Ancho en la inserción tibial, ancho en la inserción fibular, longitud del margen superior, longitud del margen inferior y el espesor. Se determinó si había contacto talar por parte del FD-LTFA y se observó desgaste articular en la superficie del domo talar, a nivel de la región de contacto del mencionado ligamento. El FD-LTFA fue encontrado en un 76,7 \% de los casos, su anchura a nivel del sitio de fijación tibial fue de 5,30 mm $( \pm 1,4)$ y a nivel fibular 4,43 mm $( \pm$ $0,85)$. En cuanto a su longitud en el margen superior fue de $14,26 \mathrm{~mm}( \pm 3,66)$ y a nivel del margen inferior fue de $16,74 \mathrm{~mm}( \pm 2,91)$. Su espesor fue de $2,1 \mathrm{~mm}( \pm 0,36)$. En las 23 muestras de tobillo con presencia del FD-LTFA, hubo contacto talar en el $100 \%$ de los casos y desgaste articular en esta zona en 3 de ellas (13,04\%). Los datos biométricos aportados por este estudio complementarán el conocimiento anatómico del FD-LTFA en la población Chilena.

PALABRAS CLAVE: Anatomía; Tobillo; Ligamento de Bassett; Ligamento tibiofibular anterior; Fascículo distal del ligamento tibiofibular anterior.

\section{INTRODUCCIÓN}

El ligamento tibiofibular anterior (LTFA) es uno de los tres ligamentos que forman la sindesmosis tibiofibular. Este ligamento es trapezoidal, plano y ancho, originándose en el tubérculo tibial anterolateral del extremo distal de la tibia (Chaput) e insertándose en el tubérculo fibular anteromedial del maléolo lateral (Wagstaffe). Se ha descrito como único o multifascicular (Bassett 3rd et al., 1990; Hermans et al., 2010; Boonthathip et al., 2010; Sarraffian, 2011; Williams et al., 2015; Lilyquist et al., 2016; Dalmau-Pastor et al., 2018).

El LTFA puede presentar un fascículo distal independiente de la banda principal, separado por una hendidura, la cual se encuentra ocupada por tejido fibroadiposo. Dicho fascículo se observa parcial o totalmente separado del LTFA. (Bassett 3rd et al.; Nikolopoulos et al., 2004, Boonthathip et al.; Hermans et al.; Dalmau-Pastor et al.).
Los textos clásicos (Testut \& Latarjet, 1972; Spalteholz, 1972; Rouvière \& Delmas, 1999; Latarjet \& Ruiz Liard, 2010; Moore et al., 2018) realizan una descripción del LTFA pero no nombran la presencia del FD-LTFA. Standring (2016) realizó una somera referencia al FD-LTFA y lo describió como una variante que se presenta bajo el LTFA, insertándose tan distalmente en la fíbula que puede causar irritación de la cara lateral del talus, sugiriendo además que es susceptible de extirpación artroscópica.

Diversos autores lo han denominado como: Ligamento tibiofibular anterior inferior accesorio (Nikolopoulos, 1982), fascículo distal del ligamento tibiofibular anterior inferior (van den Bekerom \& Raven, 2007), ligamento de Bassett (Subhas et al., 2008) y ligamento tibiofibular anterior inferior distal (Jacobson et al., 2011).

\footnotetext{
${ }^{1}$ Facultad de Medicina, Universidad Católica del Maule, Talca, Chile.

2 Programa de Magister en Ciencias, mención Morfología, Universidad de La Frontera, Temuco, Chile.

${ }^{3}$ Facultad de Medicina, Universidad de La Frontera, Temuco, Chile.
} 
Debido a que en la Terminología Anatómica Internacional (Federative Committee on Anatomical Terminology, 2011) no aparece como una entidad anatómica particular, en este estudio se le denominará fascículo distal del ligamento tibiofibular anterior (FD-LTFA).

Si bien, no todos los autores lo reconocen como una entidad independiente del LTFA (Hermans et al.), su presencia ha sido reportada entre un $21,7 \%$ (Ray \& Kriz, 1991) a $100 \%$ (Dalmau-Pastor et al.). Dicha disparidad se podría explicar por los diferentes parámetros que se utilizan para la clasificación morfológica de dicho ligamento. En este estudio, el parámetro a considerar fue la presencia de un espacio distal al LTFA, de tamaño variable, el cual puede estar ocupado por tejido fibroadiposo.

En cuanto a sus características morfológicas se han reportado longitudes medias desde los 13,1 mm (Williams et al.) a los $17 \mathrm{~mm}$ (Bartonícek et al., 2003), anchos medios desde los $3 \mathrm{~mm}$ (Boonthathip et al.) a los 4,3 mm (DalmauPastor et al.) y espesor medio entre 1,87 (Subhas et al.) y 2 mm (Dalmau-Pastor et al.).

Durante su trayecto oblicuo, las fibras más inferiores cubren el sector anterolateral de la articulación talocrural, por lo que su presencia se asocia a pellizcamiento anterolateral del tobillo, lo que resulta en un síndrome doloroso después de una lesión de inversión. Este pellizcamiento se asocia a un desgaste cartilaginoso de la cara anterolateral de la tróclea talar como consecuencia del roce del FD-LTFA en los movimientos de máxima dorsiflexión (Bassett 3rd et al.; Akseki et al., 2002; Nikolopoulos et al.; Subhas et al.). La distorsión de tobillo es una de las lesiones más comunes que se manifiesta con dolor crónico e inestabilidad. El esguince de tobillo se debe a traumatismos con inversión, flexión plantar y supinación, siendo el ligamento tibiofibular anterior, la banda más frecuentemente afectada (Seifarth, 2019).

El propósito de este estudio fue determinar presencia y biometría del FD-LTFA, y su relación con la troclea talar en tobillos de individuos Chilenos en la Región del Maule.

\section{MATERIAL Y MÉTODO}

En este estudio se utilizaron 30 miembros inferiores, de individuos Chilenos, adultos, pertenecientes a la Unidad de Anatomía Humana del Departamento de Ciencias Preclínicas de la Facultad de Medicina de la Universidad Católica del Maule, Chile. Se realizó un protocolo de disec- ción para cada muestra, y se disecaron tobillos que cumplieran con los criterios de: a) tobillos completos y b) sin lesión en el compartimento lateral de tobillo.

En las muestras ya se había disecado piel, vasos y nervios superficiales en trabajos previos, por lo que se procedió a abrir la fascia profunda de la pierna, incluyendo el retináculo extensor, para exponer el plano más profundo. Se desinsertó el músculo extensor largo de los dedos de su origen en la tibia, fíbula y membrana interósea, para retraer el músculo y exhibir la región anterior de la articulación tibiofibular distal.

Se removió el tejido adiposo, conectivo y estructuras vasculares de la zona a estudiar y se limpió el ligamento tibiofibular anterior de modo que quedó totalmente expuesto para su estudio. Se verificó la presencia del fascículo distal del ligamento tibiofibular anterior y se registró el número correspondiente a la muestra.

En posición de reposo del tobillo, se identificaron los límites del FD-LTFA y se midieron con un caliper marca Truper Stainless Steel, registrando sus valores en la tabla Excel, considerando: Ancho en la inserción tibial, ancho en la inserción fibular, longitud del margen superior, longitud del margen inferior y el espesor. Se determinó si había contacto talar por parte del fascículo distal del ligamento tibiofibular anterior.

Se realizó flexión plantar máxima para observar presencia de desgaste articular en la superficie del domo talar, a nivel de la región de contacto del fascículo distal del ligamento tibiofibular anterior.

Cada una de las muestras fue fotografiada con una cámara digital marca Nikon D7100, con marca de regla blanca de $1 \mathrm{~cm}$. Finalmente, los datos obtenidos se analizaron en el programa estadístico SPSS 17.0 para establecer media, mediana y desviación estándar.

\section{RESULTADOS}

De acuerdo a las variables observadas encontramos los siguientes resultados:

El FD-LTFA fue encontrado en 23 de los 30 tobillos correspondiendo a un 76,7 \% de los casos, estando ausente en los 7 restantes.

En relación a su anchura a nivel del sitio de fijación tibial FD-LTFA, el valor promedio fue de 5,30 $\mathrm{mm}( \pm 1,40)$, 
siendo el valor más bajo de 3,29 mm mientras que el mayor fue de $8,16 \mathrm{~mm}$ (IC95\%=4,69-5,90; $<<0,001)$.

Respecto al ancho medido en el sitio de inserción fibular del FD-LTFA, el valor promedio fue de $4,43 \mathrm{~mm}( \pm$ $0,85)$, siendo el valor más bajo de $3,05 \mathrm{~mm}$ mientras que el mayor fue de 6,75 $\mathrm{mm}$ (IC95 \%=4,06 - 4,79; $<<0,001)$.

El valor promedio de la longitud de su margen superior fue de $14,26 \mathrm{~mm}( \pm 3,66)$ con un mínimo de $8,10 \mathrm{~mm}$ y un valor máximo de 19,74 mm (IC95 \%=12,68 - 15,84; $\mathrm{p}<0,001)$.

En relación a la longitud de su margen inferior, el valor promedio fue de $16,74 \mathrm{~mm}( \pm 2,91)$, siendo el valor mínimo de $12,36 \mathrm{~mm}$ y el máximo de $23,59 \mathrm{~mm}$ (IC95 $\%=15,48-18,00 ; \mathrm{p}<0,001)$.

En relación al espesor del FD-LTFA, su valor promedio fue de $2,1 \mathrm{~mm}( \pm 0,36)$, siendo su valor mínimo de $1,46 \mathrm{~mm}$ y el máximo de 2,83 mm (IC95 \%=1,94 - 2,25; $\mathrm{p}<0,001)$.

En las 23 muestras de tobillo con presencia del FDLTFA, hubo contacto talar en el $100 \%$ de los casos y desgaste articular en esta zona en 3 de ellas (13,04\%).

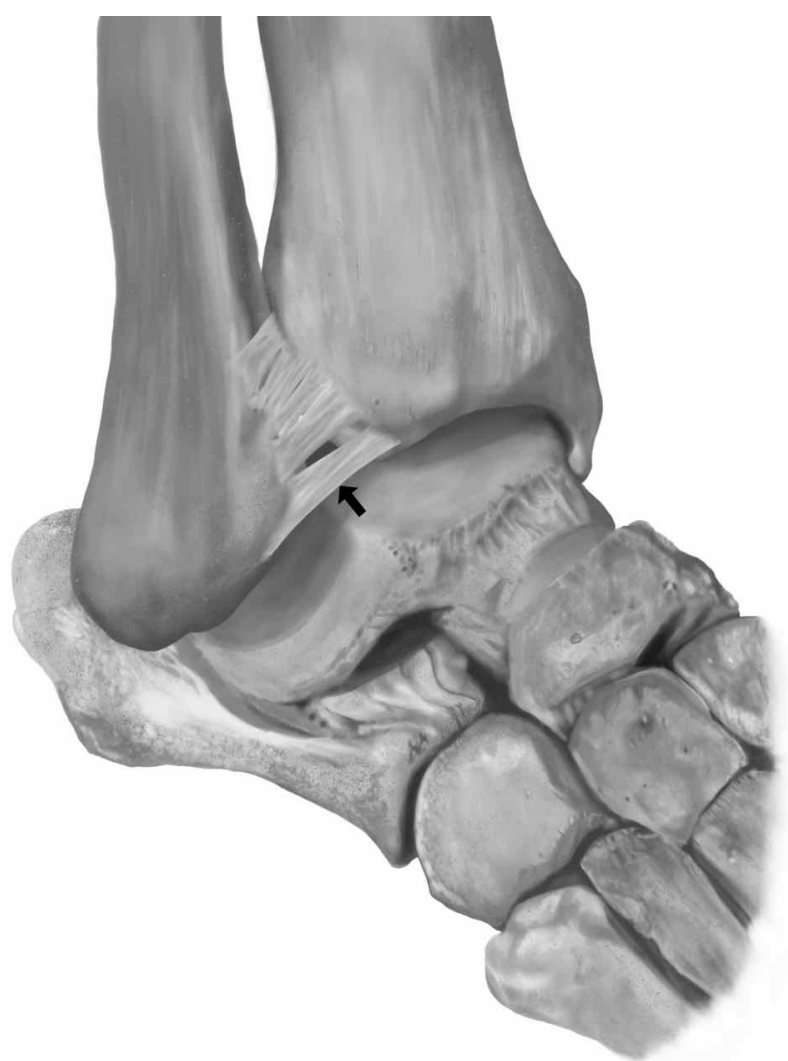

Fig. 1. Fascículo distal del ligamento tibiofibular anterior derecho (flecha).
Los 7 tobillos con ausencia del fascículo distal del ligamento tibiofibular anterior no presentaron desgaste articular. Junto con esto se realizó una prueba de Chi cuadrado con corrección por continuidad la cual no fue significativa $(\mathrm{p}=0,774)$.

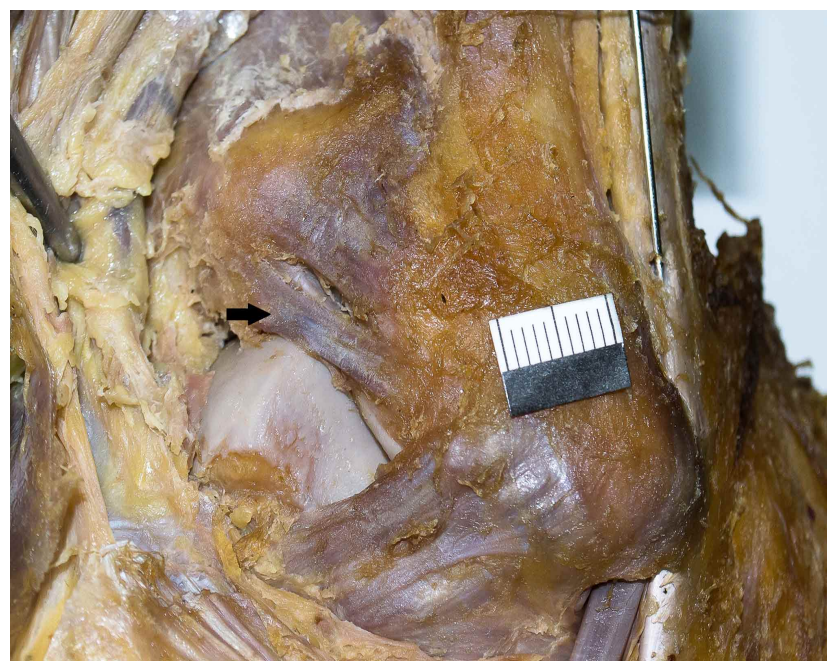

Fig. 2. Fascículo distal del ligamento tibiofibular anterior izquierdo (flecha).

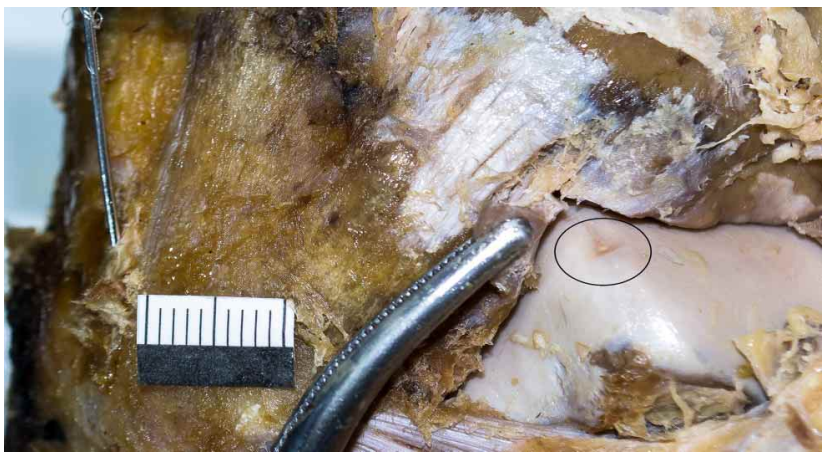

Fig. 3. Desgaste articular en la cara anterosuperior de la tróclea talar derecha en la zona de contacto con el FD-LTFA (círculo).

\section{DISCUSIÓN}

Una de las lesiones más comunes del tobillo, que se manifiesta con dolor crónico e inestabilidad es la distorsión. El esguince del mismo, se debe a traumatismos con inversión, flexión plantar y supinación, siendo el ligamento tibiofibular anterior la banda más frecuentemente afectada (Seifarth).

En el tobillo se han descrito tres tipos de lesiones intrarticulares de tejido blando las cuales incluyen hipertrofia del tejido sinovial, lesión meniscoide y pellizcamiento del FD-LTFA (van den Bekerom \& Raven; Meislin et al.,1993; Hermans et al.). 
El presente estudio reveló que la presencia del FDLTFA es alta (76,7 \%). Otros estudios en cadáveres han reportado su presencia con un número variable de muestras. Bassett 3rd et al. señalaron su presencia en un $90,9 \%$ de un total de 11 muestras. Akseki et al. informaron su presencia en un $83 \%$ de 47 muestras. Nikolopoulus et al. lo identificaron en $92 \%$ de 24 muestras y Edama et al. (2019) lo notificaron en un $87 \%$ de 100 tobillos.

También ha sido reportado un $100 \%$ de presencia del FD-LTFA (Boonthathip et al.; Williams et al.; DalmauPastor et al.) en muestras de 10, 16, 154 tobillos, respectivamente. Subhas et al. a través de imágenes de resonancia magnética, lo identificaron en un $94 \%$ de 36 exámenes.

Ray \& Kriz lo identificaron en un $21,7 \%$ de 46 muestras, porcentaje que dista radicalmente de los anteriores probablemente por el tipo de clasificación que utilizaron, considerando la presencia del FD-LTFA, sólo si se observa totalmente independiente del fascículo principal.

van den Bekerom \& Raven consideraron que la presencia del FD-LTFA probablemente es un hallazgo normal, pero no lo cuantificó. Hermans et al. proporcionaron una descripción anatómica del LTFA en adultos, mencionando que el FD- LTFA no fue observado.

Lilyquist et al. y Bartonícek et al. no informaron directamente el FD-LTFA, pero lo dividen en tercios, de los cuales, el más distal presenta las características. utilizadas en nuestro estudio y describieron su presencia en un $100 \%$ de los casos analizados.

Los datos biométricos de este estudio concuerdan con los de Dalmau-Pastor et al., ya que su ancho promedio de la fijación tibial fue de 5,1 $\mathrm{mm}$ y el del presente estudio fue de $5,3 \mathrm{~mm}$; en la fijación fibular obtuvieron $4,53 \mathrm{~mm}$ y en el presente fue de $4,43 \mathrm{~mm}$. También hubo concordancia con Boonthathip et al., quienes obtuvieron para la fijación tibial $5,3 \mathrm{~mm}$.

Estas variables fueron estudiadas también por Lilyquist et al., los que registraron un ancho promedio en la fijación tibial de $5 \mathrm{~mm}$ y en la fibular de $5,7 \mathrm{~mm}$., siendo este ultimo valor más alto que el informado en este estudio. Los valores para longitud de los márgenes superior e inferior del FD-LTBA (11,1 mm y 13,6 mm, respectivamente), fueron menores a los nuestros.

En relación al espesor del FD-LTFA, Nikolopoulus et al. y Ray \& Kriz determinaron que éste varió entre 1 a 2 $\mathrm{mm}$. por otra parte, Bartonícek et al. y Bassett $3^{\text {rd }}$ et al. informaron un espesor de $2 \mathrm{~mm}$. En estudio por imágenes,
Subhas et al. reportaron un grosor de $1,87 \mathrm{~mm}$. Estos hallazgos son concordantes con los 2,1 mm. encontrados en nuestro estudio.

Con respecto al contacto del FD-LTFA con la cara anterosuperior de la tróclea talar, nuestros resultados (100 $\%$ ), fueron concordantes con las observaciones de Bassett 3rd et al., Nikolopoulus et al. y Dalmau-Pastor et al. Valores menores, fueron encontrados por Lilyquist et al. $(89,3 \%)$ y Ray \& Kriz (70 \%). Estos resultados muestran que el contacto del FD-LTFA es una condición anatómica muy presente.

El desgaste articular en la cara anterosuperior de la tróclea talar en la zona de contacto con el FD-LTFA fue identificado en esta investigación en un 13,04 \% de las muestras analizadas, semejante a lo señalado por Akseki et al. (17 $\%)$. Resultados mayores han sido observados por Bassett 3rd et al. y Subhas et al. con un $50 \%$, Ray \& Kriz con un $82,9 \%$ y Nikolopoulus et al. en un $100 \%$.

La sola presencia del FD-LTFA no provoca un síndrome de pellizcamiento si no que depende de cambios biomecánicos en el tobillo causados por el aumento de la extrusión anterior del talus, producto del aumento de laxitud del tobillo por lesión del ligamento talofibular anterior, esto aumentaría el contacto con el FD-LTFA, acrecentando el roce con el talus (Bassett 3rd et al.).

La literatura y el presente trabajo muestra la constante presencia e importancia del FD-LTFA, sin embargo, aún no ha sido considerado como estructura independiente en la Terminologia anatomica.

Los datos biométricos aportados por este estudio complementarán el conocimiento anatómico del fascículo distal del ligamento tibiofibular anterior en la población Chilena, siendo un aporte importante a las áreas de anatomía, traumatología y quirúrgicas del tobillo.

CABEZAS, J. J. \& OLAVE, E. Distal fascicle of the anterior tibiofibular ligament: Presence, biometric characteristics and relation with the talar trochlea. Int. J. Morphol., 38(2):472-476, 2020.

SUMMARY: The anterior tibiofibular ligament usually presents an independent distal fascicle of the main band, denominated distal fascicle of the anterior tibiofibular ligament (DF-ATiFL). Which obliquely passes, covering its most inferior fiber to the anterolateral corner of the talocrural articulation. Its presence is associated with the anterolateral impingement of the ankle in this area, which can produce cartilage wear of the anterolateral surface of the talar trochlea. The purpose of this study was to determine the presence and biometry of DF-ATiFL and its 
relation with the talar trochlea on the ankles of a group of Chilean individuals. Thirty inferior members were used in this study, all from adult individuals. It was evaluated: Width of the tibial insertion, width of the fibular insertion, length of the top margin, length of the bottom margin, and the thickness. It was determined whether there was talar contact by the distal fascicle of the anterior tibiofibular ligament. Also, it was observed the articular wear on the surface of the talar dome, at the level of the contact region of the distal fascicle of the anterior tibiofibular ligament. The DFATiFL was found in $76.7 \%$ of the cases, its width at the level of the place of tibial fixation was $5.30 \mathrm{~mm}( \pm 1.40)$, and at the fibular level $4.43 \mathrm{~mm}( \pm 0.85)$. Regarding its length on the top margin was $14.26 \mathrm{~mm}( \pm 3.66)$, and at the bottom, the margin level was $16.74 \mathrm{~mm}( \pm 2.91)$. Its thickness was $2.1 \mathrm{~mm}( \pm 0.36)$. In the 23 ankles samples with the presence of DF-ATiFL, there was talar contact in $100 \%$ of the cases and articular wear in this area in 3 of them $(13.04 \%)$. The biometric data contributed by this study will supplement the anatomical knowledge of the distal fascicle of the anterior tibiofibular ligament on the Chilean population.

KEY WORDS: Anatomy; Ankle; Bassett's ligament; Anterior tibiofibular ligament; Distal fascicle of the anterior tibiofibular ligament.

\section{REFERENCIAS BIBLIOGRÁFICAS}

Akseki, D.; Pinar, H.; Yaldiz, K.; Akseki, N. G. \& Arman, C. The anterior inferior tibiofibular ligament and talar impingement: a cadaveric study. Knee Surg. Sports Traumatol. Arthrosc., 10(5):321-6, 2002.

Bartonícek, J. Anatomy of the tibiofibular syndesmosis and its clinical relevance. Surg. Radiol. Anat., 25(5-6):379-86, 2003.

Bassett 3rd, F. H.; Gates 3rd, H. S.; Billys, J. B.; Morris, H. B. \& Nikolaou, P. K. Talar impingement by the anteroinferior tibiofibular ligament. A cause of chronic pain in the ankle after inversion sprain. J. Bone Joint Surg. Am., 72(1):55-9, 1990.

Boonthathip, M.; Chen, L.; Trudell, D. J. \& Resnick, D. L. Tibiofibular syndesmotic ligaments: MR arthrography in cadavers with anatomic correlation. Radiology, 254(3):827-36, 2010.

Dalmau-Pastor, M.; Malagelada, F.; Kerkhoffs, G. M. M. J.; Karlsson, J.; Manzanares, M. C. \& Vega, J. The anterior tibiofibular ligament has a constant distal fascicle that contacts the anterolateral part of the talus. Knee Surg. Sports Traumatol. Arthrosc., 2018. doi: 10.1007/s00167018-5123-z. Online ahead of print.

Edama, M.; Takeishi, M.; Kurata, S.; Kikumoto, T.; Takabayashi, T.; Hirabayashi, R.; Inai, T.; Ikezu, M.; Kaneko, F. \& Kageyama, I. Morphological features of the inferior fascicle of the anterior inferior tibiofibular ligament. Sci. Rep., 9(1):10472, 2019.

Federative Committee on Anatomical Terminology (FCAT). Terminologia Anatomica: International Anatomical Terminology. $2^{\text {nd }}$ ed. Stuttgart, Georg Thieme Verlag, 2011.

Hermans, J. J.; Beumer, A.; de Jong, T. A. W. \& Kleinrensink, G. J. Anatomy of the distal tibiofibular syndesmosis in adults: a pictorial essay with a multimodality approach. J. Anat., 217(6):633-45, 2010.

Jacobson, K.; Ng, A. \& Haffner, K. E. Arthroscopic treatment of anterior ankle impingement. Clin. Podiatr. Med. Surg., 28(3):491-510, 2011.

Latarjet, M. \& Ruiz Liard, A. Anatomía Humana. $4^{\mathrm{a}}$ ed. Buenos Aires, Médica Panamericana, 2010.

Lilyquist, M.; Shaw, A.; Latz, K.; Bogener, J. \& Wentz, B. Cadaveric analysis of the distal tibiofibular syndesmosis. Foot Ankle Int., 37(8):882-90, 2016
Meislin, R. J.; Rose, D. J.; Parisien, J. S. \& Springer, S. Arthroscopic treatment of sinovial impingement of the ankle. Am. J. Sports Med., 21(2):186-9, 1993.

Moore, K.; Dalley, A.; Agur, A.; Gutiérrez, A. \& Ángeles, C. Anatomía con Orientación Clínica, $8^{\mathrm{a}}$ ed. Barcelona, Wolters Kluwer, 2018.

Nikolopoulos, C. Anterolateral instability of the ankle joint: an anatomical, experimental, and clinical study. Tesis. Atenas, University of Athens, 1982.

Nikolopoulos, C. E.; Tsirikos, A. I.; Sourmelis, S. \& Papachristou, G. The accessory anteroinferior tibiofibular ligament as a cause of talar impingement: a cadaveric study. Am. J. Sports Med., 32(2):389-95, 2004.

Ray, R. G. \& Kriz, B. M. Anterior inferior tibiofibular ligament. Variations and relationship to the talus. J. Am. Podiatr. Med. Assoc., 81(9):479$85,1991$.

Rouvière, H. \& Delmas, A. Anatomía Humana, Descriptiva, Topográfica y Funcional. 11 a ed. París, Masson, 1999.

Seifarth, A. Anatomie und pathologie der bänder im oberen und unteren sprunggelenk. Arthroskopie, 32(2):125-32, 2019.

Spalteholz, W. Atlas de Anatomía Humana. 6ª ed. Barcelona, Labor, 1972.

Standring, S. Gray's Anatomy. The Anatomical Basis of Clinical Practice. $41^{\mathrm{a}}$ ed. New York, Elsevier, 2016.

Subhas, N.; Vinson, E. N.; Cothran, R. L.; Santangelo, J. R.; Nunley 2nd, J. A. \& Helms, C. A. MRI appearance of surgically proven abnormal accessory anterior-inferior tibiofibular ligament (Bassett's ligament). Skeletal Radiol., 37(1):27-33, 2007.

Testut, L. \& Latarjet, A. Tratado de Anatomía Humana. $9^{\text {a }}$ ed. Barcelona, Salvat, 1972

van den Bekerom, M. P. J. \& Raven, E. E. J. The distal fascicle of the anterior inferior tibiofibular ligament as a cause of tibiotalar impingement syndrome: a current concepts review. Knee Surg. Sports Traumatol. Arthrosc., 15(4):465-71, 2007.

Williams, B. T.; Ahrberg, A. B.; Goldsmith, M. T.; Campbell, K. J.; Shirley, L.; Wijdicks, C. A.; LaPrade, R. F. \& Clanton, T. O. Ankle syndesmosis: a qualitative and quantitative anatomic analysis. Am. J. Sports Med., 43(1):88-97, 2015.

Dirección para correspondencia:

Prof. Dr. Enrique Olave

Facultad de Medicina

Universidad de La Frontera

Av. Francisco Salazar 01145

Temuco

CHILE

Email: enrique.olave@ufrontera.cl

Recibido : 20-10-2019

Aceptado: 21-11-2019 